\title{
Disconnection of the Hippocampal-Perirhinal Cortical Circuits Severely Disrupts Object-Place Paired Associative Memory
}

\author{
Yong Sang Jo ${ }^{1}$ and Inah Lee ${ }^{2}$ \\ ${ }^{1}$ Department of Psychology, University of Washington, Seattle, Washington 98195, and 2Department of Brain and Cognitive Sciences, Seoul National \\ University, Seoul 151-742, Korea
}

The hippocampus and the perirhinal cortex (PR) are reciprocally connected both directly and indirectly via the entorhinal cortex. Although it has been hypothesized that the two regions should have intimate functional interactions with each other on the basis of the anatomical connectivity, many lesion studies have demonstrated functional dissociations instead between the hippocampus and PR. To show a tight functional relationship between the two regions, we used reversible inactivation techniques targeting both the hippocampus and PR within subjects, combined with a biconditional memory task in which the rat must consider information about objects and their locations. Specifically, rats were implanted with two sets of bilateral cannulas into the hippocampus and PR, and were tested in an object-place paired-associate task in a radial maze. While alternating between two arms, the rats were required to choose one of the objects exclusively associated with a given arm for food. Bilateral muscimol (MUS) injections into either the hippocampus or PR equally produced chance level performance. When a functional disconnection procedure was used to disrupt the interaction between the hippocampus and PR, contralateral MUS injections into the hippocampus and PR resulted in severe impairment in performance. However, inactivating the hippocampus and PR ipsilaterally did not affect the performance. In a simple object discrimination task, the same functional disconnection protocol with MUS did not affect the performance. The results powerfully demonstrate that the hippocampus, the $\mathrm{PR}$, and their functional interactions are all indispensable when objects and their spatial locations must be processed at the same time.

\section{Introduction}

The hippocampus (HP) and the perirhinal cortex (PR) in the medial temporal lobe (MTL) are densely connected both directly and indirectly via the entorhinal cortex (EC) (Witter et al., 1989, 2000; Burwell et al., 1995). The reciprocal connections between the two areas suggest that the PR should heavily influence the information processing in the hippocampus and vice versa. However, previous studies overall suggest that the hippocampus and PR can be functionally dissociated (Ennaceur et al., 1996; Mumby, 2001; Aggleton et al., 2004; Winters et al., 2004; Aggleton and Brown, 2005; Saksida et al., 2006; Balderas et al., 2008; Abe et al., 2009). The overall consensus from those studies is that the hippocampus is essential for spatial memory and the PR is important for object recognition.

The functional contrast between the two regions suggested in the literature serves its purpose for uncovering multiple memory systems in the MTL. However, it has failed to demonstrate possible functional interactions between the hippocampus and PR, which has been suggested by a leading theoretical framework for

Received March 28, 2010; revised May 3, 2010; accepted June 14, 2010.

This work was supported by the World Class University Program of the Ministry of Education, Science and Technology in Korea through Korea Science and Engineering Foundation Grant R32-10142 and by National Institute of Mental Health Grant R01 MH079971.

Correspondence should be addressed to Dr. Inah Lee, Department of Brain and Cognitive Sciences, Seoul National University, Gwanak-ro, Gwanak-gu, Seoul 151-742, Korea. E-mail: inahlee@snu.ac.kr.

DOI:10.1523/JNEUROSCI.1580-10.2010

Copyright $\odot 2010$ the authors $\quad 0270-6474 / 10 / 309850-09 \$ 15.00 / 0$ understanding the hippocampal function in encoding and retrieving event memory (Davachi, 2006; Knierim et al., 2006; Eichenbaum and Lipton, 2008). Specifically, parallel information processing streams for object and space have been implicated in the MTL by the anatomical literature (Burwell, 2000; Witter et al., 2000; Witter and Amaral, 2004). According to this view, object information is processed by the $\mathrm{PR}$ and is transmitted to the lateral entorhinal cortex (LEC) before reaching the hippocampus, whereas spatial information is sent to the hippocampus via a different route involving the postrhinal cortex (PoR) and the medial entorhinal cortex (MEC). Since the information for both object and place should be channeled reliably to the hippocampus to be combined as a unitary event, the hippocampus and its upstream structures (e.g., PR, PoR, LEC, and MEC) should be equally important for the representation of an object-place associative event. Therefore, a task that maximally taps into the integrity of such a system in object-place association should predict severe functional impairment when either the hippocampus or any of its upstream structures is disrupted. Few studies have addressed this issue by directly manipulating both the hippocampus and the PR using a memory task that places a heavy demand on processing object-place paired-associative information. For example, Gaffan and Parker (1996) used an object-in-place task in monkeys but they manipulated the fimbria-fornix instead of directly targeting the hippocampus and lesion methods were used compared with reversible inactivation techniques for examining normal functions of the two areas. 
Based on the previous results from our laboratory (Lee and Solivan, 2008; Jo and Lee, 2010), it was predicted that bilateral inactivations of either the hippocampus or PR would impair performance in the task equally. The current study further aims to examine whether the tight interactions between the hippocampus and perirhinal cortex are necessary for the object-place paired-associate task using reversible inactivation techniques targeting both the hippocampus and PR.

\section{Materials and Methods}

Subjects. Male Long-Evans rats $(n=8)$ weighing between 320 and $380 \mathrm{~g}$ were housed individually in Plexiglas cages in a temperature- and humidity-controlled environment. All animals were maintained on a $12 \mathrm{~h}$ light/dark cycle, and all behavioral experiments were conducted during light phase of the cycle. Each rat was allowed access to water ad libitum and food-deprived to $80 \%$ of its free-feeding weight for behavioral testing.

Behavioral apparatus. Detailed information of the apparatus can be found in our previous study (Lee and Solivan, 2008, 2010; Jo and Lee, 2010). Briefly, a radial-arm maze made of black Plexiglas contained seven arms $(10 \times 80 \mathrm{~cm}$ each $)$ radiating from a circular center stage $(48 \mathrm{~cm}$ in diameter) (Fig. $1 \mathrm{~A})$. A rectangular choice platform $(23 \times 30 \mathrm{~cm})$ at the end of each arm had three food wells separated from each other by transparent vertical dividers. A transparent guillotine door $(10 \times 25 \mathrm{~cm})$ was placed at the entrance of each arm to allow access to the arm. A start box $(20 \times 25 \times 30 \mathrm{~cm})$ with a black guillotine door was located in the center stage, although it was not used for the current task. Only two arms of the maze were used and a black Plexiglas panel $(90 \times 40 \mathrm{~cm})$ was placed in the center arm to block the rat's view of the choice platform on the other side (Fig. 1A). The maze was surrounded by black curtains and the curtains were decorated with several distinctive visual cues. A digital CCD camera was positioned above the maze for recording behavioral experiments, and two loud speakers were placed underneath the center stage to provide white noise during the experiments.

Object-place paired-associate task. The procedures on behavioral training were the same as in our previous study (Lee and Solivan, 2008, 2010; Jo and Lee, 2010) except that rats alternated between two arms in the maze without entering the start box. Briefly, naive rats were initially trained to obtain a quarter piece of Froot Loops cereal (Kellogg's) from a middle food well of the choice platform fully covered with a wooden block in a separate room. After introduced to the maze, each rat was placed on one of the choice platforms of two arms (named arm 3 and arm 5 clockwise) (Fig. $1 A$ ) and was trained to continuously alternate between the two arms to collect rewards. The doors for the two arms were left open throughout the experiment. Once the rat finished 32 alternation trials in this simple task within $30 \mathrm{~min}$, an object-place paired-associate task started.

For the object-place paired-associate task, either arm 3 or 5 was selected randomly at the beginning of the session and two food wells (left and right food wells; the middle food well was never used in the task) of the choice platform were completely covered by two toy objects (named Boy and Potatohead; 6.5 and $5 \mathrm{~cm}$ tall, respectively). The two objects were selected because rats in our laboratory did not show any preference for either object previously. Then, the rat was placed on the choice platform (with no objects) of the other arm with a heavy metal block $(10 \times 28$ $\mathrm{cm}$ ) positioned in the junction between the arm and the choice platform to prevent the animal from exiting the choice platform until the experimenter removed the block. A rectangular opaque panel $(50 \times 70 \mathrm{~cm})$ was placed between arms 3 and 5 (Fig. 1A) so that rats could not see the objects arranged in the choice platform in the opposite arm. Once the objects were prepared in the target choice platform, the rat was allowed to leave the current arm as the experimenter removed the metal block. The rat ran down and exited the arm and entered the target arm via the center stage. The rat encountered the two objects at the end of the target arm and, to obtain a cereal reward, should learn to displace a particular object associated with reward in a given arm (Boy in arm 3 and Potatohead in arm 5) regardless of the location (left or right food well) of the rewarding object within the choice platform. The locations of the objects in a given choice platform were counterbalanced across trials. Once the rat made a correct choice by displacing one of the objects, the experimenter placed the metal block again in the entrance of the choice platform to prevent the animal from exiting the arm voluntarily. The rat was allowed to eat the cereal reward once the choice was correct but a correction choice was not allowed once the animal made a wrong choice. (The experimenter blocked the animal with a small Plexiglas panel and removed the reward in the food well with the two objects from the choice platform.) While the rat was confined in the choice platform, the experimenter prepared for the next trial by arranging the two objects in the choice platform in the other arm.

Thirty-two trials were given per day with an intertrial interval of 20-30 s. In addition to the percent correct score, the following variables were also measured off-line: (1) the latency from exiting a given arm to displacing an object in the other choice platform, (2) the number of spatial working memory (SWM) errors caused by reentering the arm visited in the previous trial, and (3) the perseverative response pattern measured by a response bias index calculated by taking an absolute value of the result from the following formula: (no. left choice - no. right choice)/(no. left choice + no. right choice), where no. left choice or no. right choice indicates the number of left or right choices. When a SWM error was made within a trial, the duration between the last exit time of the previously visited arm and the displacement of an object was taken as latency. After being trained to criterion ( $\geq 75 \%$ correct choices for both arms for 2 consecutive days), each rat received surgery (see below). Among the nine rats that initially started training in the task, one of the animals showed unusually slow learning compared with the other eight rats and that rat was excluded from the study. It took 9-15 d (median, $10.5 \mathrm{~d}$ ) for the remaining eight rats to learn the task to criterion before surgery.

Surgery. Each rat was deeply anesthetized under isoflurane (4\% mix with oxygen at a flow rate of $1 \mathrm{~L} / \mathrm{m}$ ) in an induction chamber, followed by intraperitoneal injection of ketamine $(60 \mathrm{mg} / \mathrm{kg})$ and xylazine $(8 \mathrm{mg} / \mathrm{kg})$. The animal was placed in a stereotaxic instrument (Stoelting). The anesthesia was maintained by isoflurane (1-3\%) afterward throughout surgery. The skull was exposed and adjusted to place bregma and lambda on the same horizontal plane. After small burr holes were drilled, two sets of 26 gauge guide cannulas (Plastics One) were implanted bilaterally into the dorsal hippocampus (HP group; $3.8 \mathrm{~mm}$ posterior to bregma, 2.6 $\mathrm{mm}$ lateral to midline, $3.5 \mathrm{~mm}$ ventral from the skull surface) and the PR (PR group; $4.8 \mathrm{~mm}$ posterior to bregma, $7.6 \mathrm{~mm}$ lateral to midline at an angle of $10^{\circ}$ with the tip oriented medially, $3.9 \mathrm{~mm}$ ventral from the skull surface). The cannulas were secured in place with anchoring screws and dental cement. A 32 gauge dummy cannula was inserted into each cannula to prevent clogging. Rats were allowed to recover for $7 \mathrm{~d}$, during which they were handled daily. All protocols conformed to the National Institutes of Health Guide for the Care and Use of Laboratory Animals and the Institutional Animal Care and Use Committee.

Intracranial microinjection. Muscimol (MUS) $(0.2 \mu \mathrm{g} / 0.2 \mu \mathrm{l})$, a $\mathrm{GABA}_{\mathrm{A}}$ receptor agonist, was used to temporally inactivate the hippocampus and PR. On injection days, after the dummy cannulas were removed, a 33 gauge injection cannula extending $1 \mathrm{~mm}$ below the tip of the guide cannula was inserted. The injection cannula was backfilled with mineral oil and connected to a $10 \mu \mathrm{l}$ syringe (Hamilton). Either MUS or saline (SAL) was injected at a rate of $10 \mu \mathrm{l} / \mathrm{h}$ using a microinfusion pump (KD Scientific). The injection quantity was $0.3 \mu \mathrm{l} /$ side for the hippocampus and $0.5 \mu \mathrm{l} /$ side for the PR. The injection cannula was left in place for an additional $1 \mathrm{~min}$ to achieve a proper diffusion of the drug from its tip. The rats were then returned to their home cages, and any abnormality in movement was examined for $30 \mathrm{~min}$ before they were placed in the maze.

Behavioral testing after surgery. To investigate the effects of inactivation of the hippocampus and PR, a within-subjects design was used throughout the experiments. Specifically, after a week of recovery, the rats were retrained to criterion in the object-place paired-associate task. Once each rat reached criterion, on the next day, either SAL or MUS was injected bilaterally into the HP for 4 consecutive days with the following schedule: day 1, SAL; day 2, MUS; day 3, MUS; day 4, SAL (Fig. $1 B$ ). After the hippocampal-inactivation schedule was completed, a bilateral injection schedule for the PR began with the same protocol used for the hippocampal injections described above (i.e., SAL-MUS-MUS-SAL). Afterward, 
the interaction between the hippocampus and PR was tested as follows. The rat was tested in the task after either SAL or MUS was injected into the hippocampus on one hemisphere with the same drug (SAL or MUS) injected into the PR on the contralateral side (HP/conPR) (e.g., the hippocampus in the right hemisphere and the $\mathrm{PR}$ in the left hemisphere as shown in Fig. $1 B$ ) for 4 consecutive days (SAL-MUS-MUS-SAL). The left/right hemisphere that received the drug injection was counterbalanced among the subjects. In addition, the effects of unilateral injection of either SAL or MUS into both the hippocampus and $\mathrm{PR}$ of the same hemisphere (HP/ ipsiPR) (e.g., the hippocampus and PR in the left hemisphere) (Fig. $1 B$ ) were also evaluated for the next 4 consecutive days. The left/right hemisphere that received the drug injections was counterbalanced among the rats. On the last day of the task, MUS was injected unilaterally into the $\mathrm{HP} /$ conPR again to test a possible effect of prolonged behavioral testing under drug infusion on performance.

Simple object discrimination task. After testing the animals in the main task, the rats were trained and tested for additional $6 \mathrm{~d}$ to determine whether the interaction between the hippocampus and PR was necessary in a simple object discrimination task in which the rats were not required to process object and spatial information at the same time (Fig. 1C). Specifically, two different pairs of objects were used by adding a different pair of objects (Lego girl and Cylinder; 4.5 and $4.6 \mathrm{~cm}$ tall, respectively) to the original pair and a given pair of the objects was presented only on either arm 3 or arm 5 . While alternating between the two arms, rats were required to choose a certain object for a cereal reward in arm 3 (Boy) and arm 5 (Cylinder) regardless of the location of the rewarding object within the choice platform (Fig. 1C). Left and right positions of the objects were counterbalanced between trials as shown in Figure 1C. Thirty-two trials were given per day with an intertrial interval of 20-30 s. Once correct performance of each rat was $\geq 75 \%$ for both arms for 2 consecutive days, either SAL or MUS was injected contralaterally into the HP/ conPR for $4 \mathrm{~d}$ with the same drug injection schedule (SAL-MUS-MUS-SAL) used in the objectplace paired-associate task.

Histology. After the completion of all behavioral experiments, cannula positions were verified histologically. To estimate the site of drug injection and approximate range of spread of the drug in the hippocampus and the PR, fluorescent MUS (BODIPY TMR-X muscimol; Invitrogen) was injected into the hippocampus and PR 30 min before anesthesia. All rats received a lethal dose of sodium pentobarbital (Euthasol; Virbac $\mathrm{AH})$, followed by a transcardial infusion of $0.9 \%$ saline and a 10\% formaldehyde solution. Each brain was stored in a $10 \%$ formalin-30\% sucrose solution at $4^{\circ} \mathrm{C}$ for $72 \mathrm{~h}$. The brains were frozen and cut in coronal sections $(40 \mu \mathrm{m})$ on a sliding microtome (Microm HM 430; Thermo Fisher Scientific). The sections then were mounted on gelatin-coated slides, stained for Nissl with thionin (Sigma-Aldrich), and examined under light microscopy. The sections labeled with the fluorescent MUS were assessed using a yellow-orange fluorescent filter (572 $\mathrm{nm}$ wavelength).
A

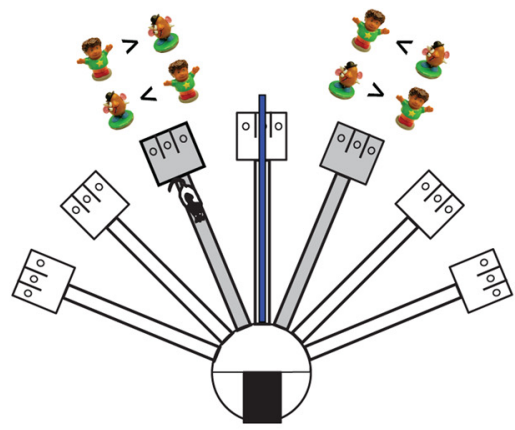

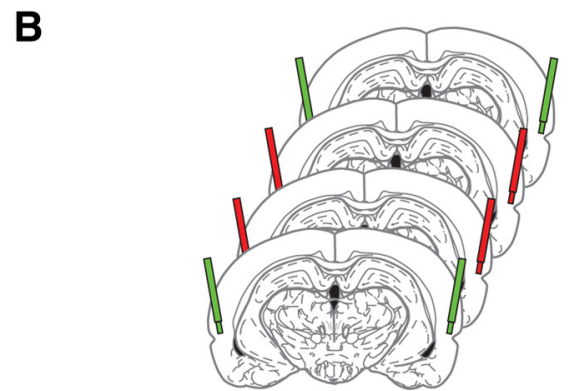

PR

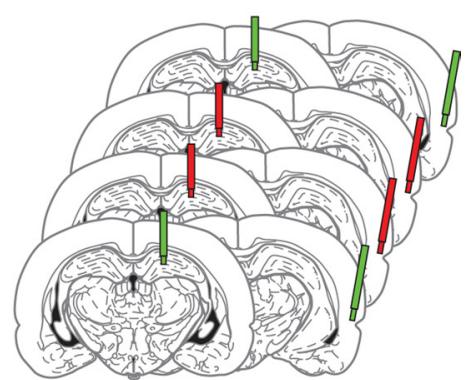

HP/ipsiPR

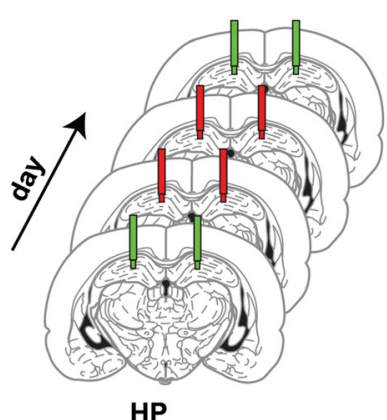

HP

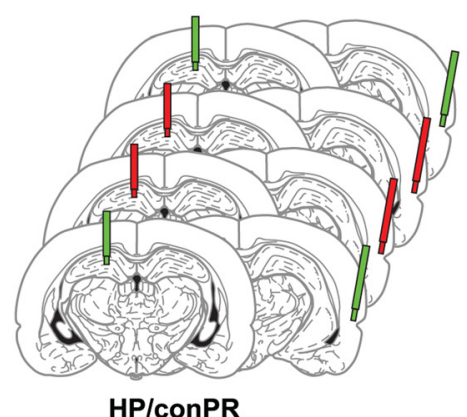

HP/conPR

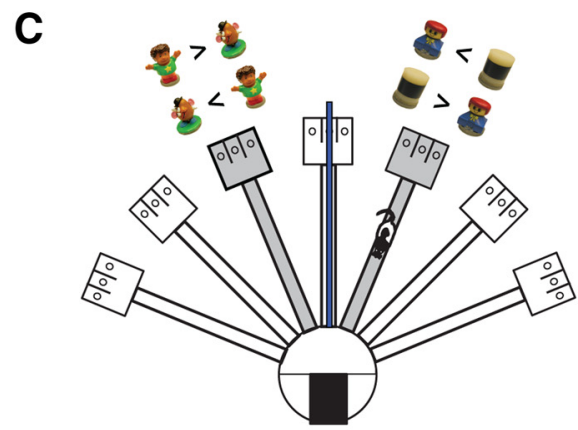

Figure 1. Behavioral paradigm and inactivation methods. $\boldsymbol{A}$, Illustration of the radial maze for the object-place pairedassociate task. A pair of objects (Boy and Potatohead) was presented in arm 3 and arm 5 . While alternating between the two arms, rats were required to choose a certain object for reward in arm 3 (Boy) and arm 5 (Potatohead). Possible configurations of objects and appropriate choices are provided for both arms for illustrative purposes. The blue bar in the center arm illustrate the black pane used to block the rat's view from one arm for the other arm. $\boldsymbol{B}$, Illustration of drug injection schedules. The green cannulas indicate SAL injections, and the red cannulas denote MUS injections (the cannulas not used for injection in certain conditions are not shown for illustrative purposes only). Each rat underwent the following injection schedule: SAL-MUS-MUS-SAL in the HP (4 d), SALMUS-MUS-SAL in the PR (PR; 4 d), SAL-MUS-MUS-SAL in the contralateral hippocampus-PR (HP/conPR; 4 d), and SAL-MUSMUS-SAL in the ipsilateral hippocampus-PR (HP/ipsiPR; 4 d). Only HP/conPR and HP/ipsiPR conditions involving the left PRs are shown for illustrative purposes. $C$, Illustration of the simple object discrimination task. The testing paradigm was same as the object-place paired-associate task shown in $\boldsymbol{A}$, except that different pairs of objects were presented in arms 3 and 5 .

\section{Results}

\section{Histology}

The cannula positions for all animals are shown in Figure 2. The tips of all injection cannulas were located within the hippocam- 
A
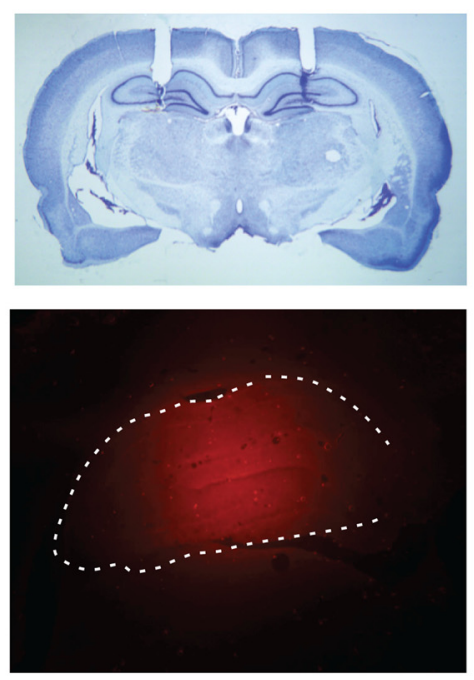

B

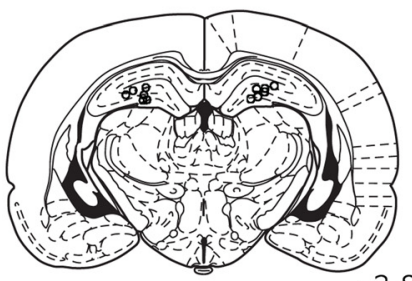

$-3.8 \mathrm{~mm}$

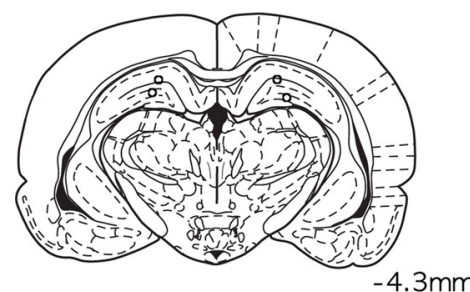

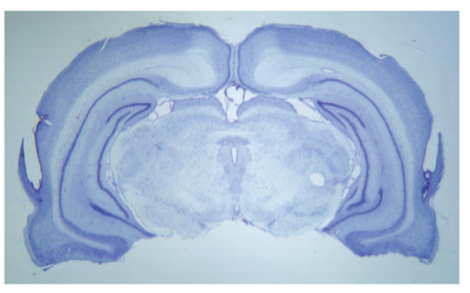
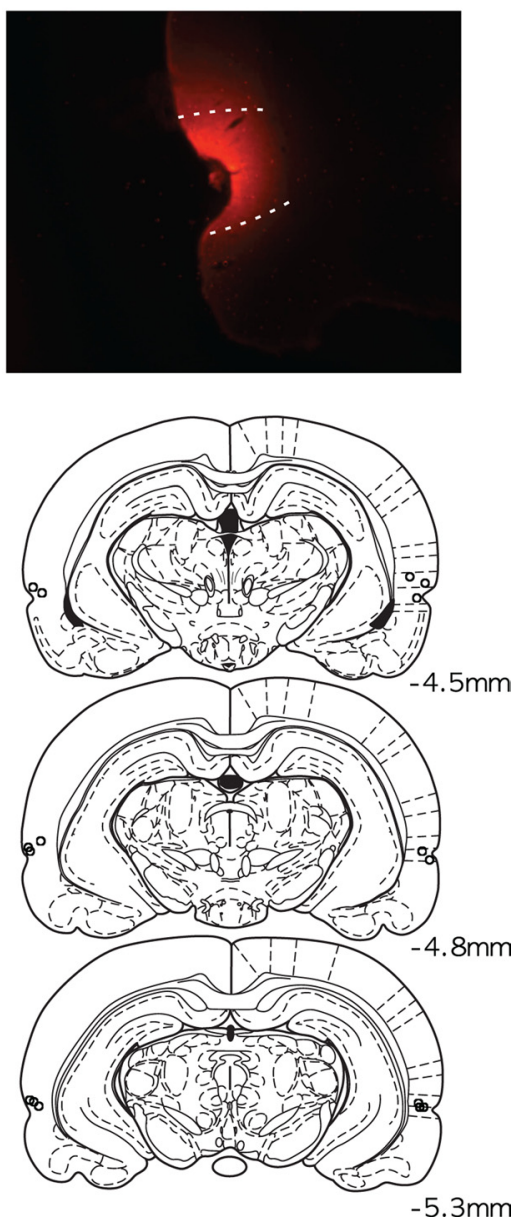

$-5.3 \mathrm{~mm}$

Figure 2. Histological verification of cannula positions and drug injection sites. $A$, Representative photomicrographs for cannula placement in the hippocampus (left) and PR (right). The top panel shows Niss--stained sections, and the bottom panel displays the spread of fluorescent MUS. The dotted lines in the bottom panel show the boundaries of the hippocampus (left) and the PR (right). $\boldsymbol{B}$, Illustration of the locations of the injection cannula tips in the hippocampus and PR in all animals used in the study. The numbers show distances from bregma.

pus and PR as shown in Figure 2, $A$ and $B$. The spread of fluorescent MUS was well localized in the target areas (Fig. 2A; supplemental Fig. 1, available at www.jneurosci.org as supplemental material). Considering that the brain was extracted $30 \mathrm{~min}$ after the injection of fluorescent MUS, these results verified that the MUS inactivations were fairly localized in the hippocampus and PR when the rats were tested on the maze after the drug infusion.

\section{Effects of the bilateral inactivation of the hippocampus and PR on object-place paired association}

After the rats were trained in the task, either SAL or MUS was injected bilaterally into the hippocampus. The rats showed $\sim 90 \%$ correct performance with the first SAL injections (Fig. $3 A$ ). The performance dropped markedly, however, almost to chance level when MUS was injected for $2 \mathrm{~d}$ consecutively. When SAL was injected again on the next day, the rats regained the previous level of performance. An ANOVA with repeated measures revealed a significant effect of drug injection day $\left(F_{(3,21)}=\right.$ $103.3 ; p<0.001)$. Post hoc pairwise comparisons (Bonferroni's $t$ test) demonstrated significant differences in correct performance between the first SAL and MUS injections during the first $2 \mathrm{~d}(p<0.001)$ and between the MUS and SAL injections in the last $2 \mathrm{~d}(p<0.001)$. No significant differences were found between the SAL injections on days 1 and $4(p>0.9)$ or between the MUS injections on days 2 and $3(p>0.9)$. The results strongly suggest that the hippocampus is necessary in the current task and confirm our previous findings (Lee and Solivan, 2008, 2010).

Then, the effects of the bilateral inactivations of the PR were tested in the following 4 d. Bilateral injections of MUS induced profound decrease in performance, whereas SAL injections before and after MUS injections did not affect the performance. An ANOVA with a repeatedmeasures design showed a significant effect of drug injection day $\left(F_{(3,21)}=\right.$ 125.6 ; $p<0.001)$. Post hoc pairwise comparisons (Bonferroni's $t$ test) revealed that the animals made significantly more errors during the $2 \mathrm{~d}$ of MUS injections than during those of SAL injections (values of $p<0.001)$. No significant differences were found between the SAL injections on days 5 and $8(p>0.9)$ or between the MUS injections on days 6 and $7(p>0.9)$. Moreover, an ANOVA with repeated measures showed no difference in behavioral performance among MUS injection days (i.e., days 2, 3, 6, and 7) for the hippocampus and $\operatorname{PR}\left(F_{(3,21)}=0.49 ; p=\right.$ $0.69)$, suggesting that the impairment produced by the PR inactivation was equivalent to the deficits observed with the hippocampal inactivation in the object-place paired-associate task. These results strongly demonstrate that not only the hippocampus but also the PR is necessary in the current object-place paired-associate task, confirming our previous lesion study (Lee and Solivan, 2008; Jo and Lee, 2010).

The latency from exiting a given arm to displacing an object in the next target arm was measured to examine whether MUS injections caused generic sensory-motor deficits that might have affected the performance of the rats in the current task (Fig. 3B). A paired $t$ test was performed on the latency data (averaging $2 \mathrm{~d}$ of SAL and MUS conditions), and the results showed that there was no difference between SAL and MUS injections into the hippocampus $\left(t_{(15)}=0.46 ; p=0.64\right)$ and the $\operatorname{PR}\left(t_{(15)}=\right.$ $0.29 ; p=0.77)$.

Effects of contralateral and ipsilateral disconnection between the hippocampus and PR

The bilateral inactivation study showed that both the hippocampus and PR are necessary for the task. To further investigate 

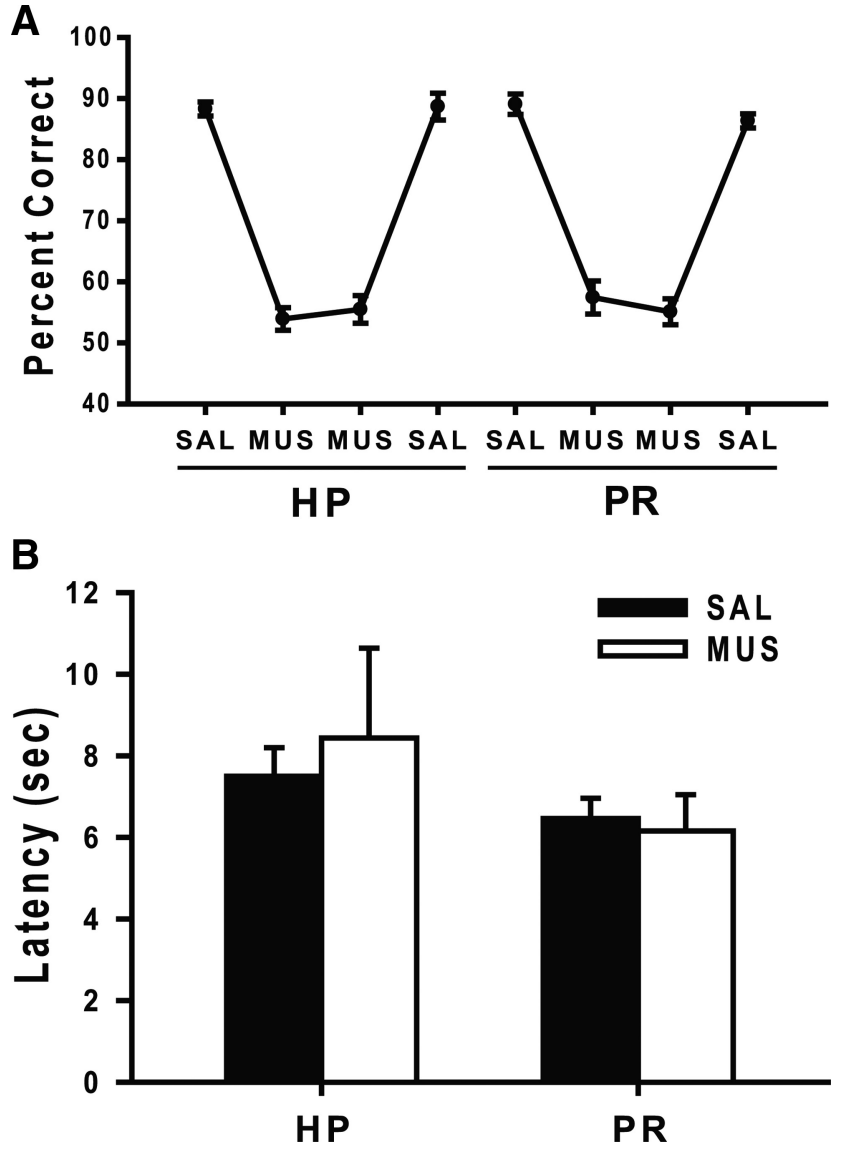

Figure 3. Effects of bilateral inactivations of the HP and PR on the performance in the object-place paired-associate task. $A$, Percentage correct performance with either SAL or MUS injections into the hippocampus and PR bilaterally. Both HP and PR inactivations produced severe deficits in performance. $\boldsymbol{B}$, Average latency from starting arms to object choice during behavioral testing. No significant difference in latency was found with MUS injections compared with SAL-injected conditions. All graphs show mean \pm SEM.

whether the interaction between the two regions is also necessary in the current task, either SAL or MUS was injected into the hippocampus of one hemisphere and the same drug was injected contralaterally into the PR for 4 consecutive days (Fig. $1 \mathrm{~B}, \mathrm{HP} /$ conPR). This procedure disallows the most dominant interactions between the two regions because the CA1 in the hippocampus and the ipsilaterally located PR exhibit heavier reciprocal projections than the CA1 and the contralaterally located PR (Witter and Amaral, 2004). Rats injected with SAL into the HP/conPR maintained $>90 \%$ correct performance in the first and last injection days (Fig. 4A). On the contrary, the animals injected with MUS into the HP/conPR exhibited chance-level performances for $2 \mathrm{~d}$ consecutively. An ANOVA with a repeated-measures design showed a significant effect of drug injection day $\left(F_{(3,21)}=102.4 ; p<0.001\right)$. Post hoc pairwise comparisons (Bonferroni's $t$ test) revealed that there were significant differences between SAL and MUS injections in the first $2 \mathrm{~d}(p<0.001)$ and between the following MUS and SAL injections in the last $2 \mathrm{~d}$ $(p<0.001)$.

To confirm that the above results were attributable to the lack of the dominant interaction between the ipsilateral hippocampus and PR, the effects of the ipsilateral injections of SAL or MUS were examined by injecting either SAL or MUS into the hippocampus and PR on the same hemisphere (Fig. $1 B, \mathrm{HP} / \mathrm{ipsiPR}$ ). This procedure should allow normal interactions between the hippocampus and PR on one hemisphere. The SAL injections in
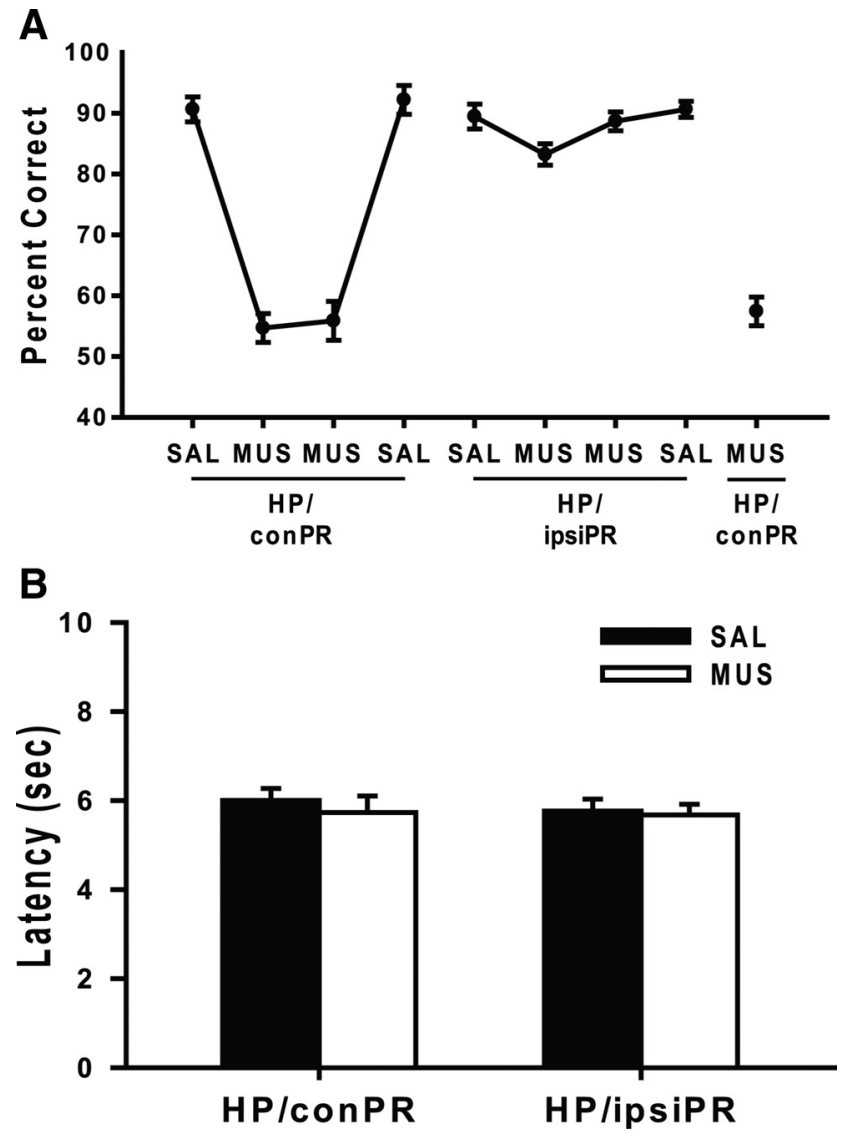

Figure 4. Disconnection between the hippocampus and PR in the object-place pairedassociate task. $\boldsymbol{A}$, Effects of either contralateral (HP/conPR) or ipsilateral (HP/ipsiPR) disconnection between the hippocampus and PR on performance. Profound deficits were found with the $\mathrm{HP} / \mathrm{conPR}$ inactivations but not in the HP/ipsiPR condition. $\boldsymbol{B}$, Average latency from starting arms to object choice during behavioral testing. No significant difference in latency was found in any of the disconnection conditions. All graphs show mean \pm SEM.

the HP/ipsiPR condition exerted no influence on behavioral performance, whereas the rats showed slight impairment with the first injection of MUS although they quickly improved to the normal level of performance in the second day (Fig. 4A). An ANOVA with repeated measures revealed a significant effect of drug injection day $\left(F_{(3,21)}=8.79 ; p=0.001\right)$, which was likely attributable to the highly consistent performances in the first and the last $2 \mathrm{~d}$ in the HP/ipsiPR condition. Post hoc pairwise comparisons (Bonferroni's $t$ test) demonstrated that the performance on the first day of MUS injection was lower than the performance on the second MUS injection and the last SAL injection condition (values of $p<0.05$ ). However, there was no significant difference in performance between the first $2 \mathrm{~d}$ of SAL and MUS injections $(p=0.09)$. Furthermore, an ANOVA with repeated measures revealed that the behavioral performance of the HP/conPR inactivation was significantly lower than that of the $\operatorname{HP} /$ ipsiPR $\left(F_{(3,21)}=\right.$ 133.1; $p<0.001)$.

To test a possibility that the HP/ipsiPR inactivation resulted in the improved performance because the rats developed some tolerance to MUS across multiple days of testing, the HP/conPR was inactivated again with MUS. The MUS injection into the HP/ conPR caused a sharp drop in performance again to $<60 \%$ (Fig. $4 A$ ). An ANOVA with a repeated-measures design showed that this performance under MUS inactivation was significantly lower than the performances associated with the MUS injections in the $\mathrm{HP} / \operatorname{ipsiPR}\left(F_{(2,14)}=143 ; p<0.001\right)$. As shown in Figure $4 B$, no 


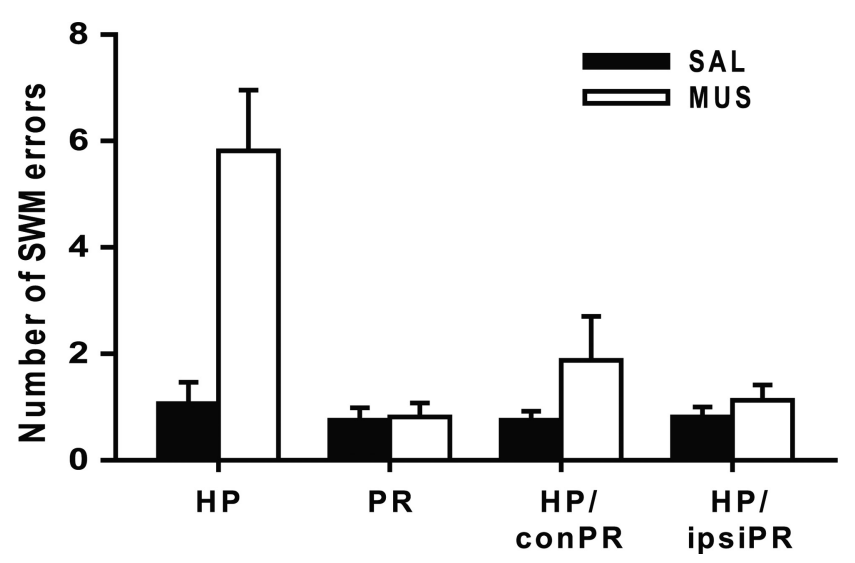

Figure 5. Total number of SWM errors made by rats ( $2 \mathrm{~d}$ of data were grouped into a block) with either SAL or MUS injections. Only the bilateral injections of MUS in the hippocampus produced significantly increased SWM errors. All graphs show mean \pm SEM.

difference was found in the latency ( $2 \mathrm{~d}$ grouped into a block) between SAL and MUS injections in both the HP/conPR $\left(t_{(15)}=\right.$ $0.62 ; p=0.53)$ and HP/ipsiPR conditions $\left(t_{(15)}=0.38 ; p=0.7\right)$.

Overall, the disconnection paradigm used in the current study strongly demonstrates that the ipsilateral interactions are very important between the hippocampus and the PR for the rats to perform normally in the current object-place paired-associate task, whereas the contralateral interactions between the two regions contribute minimally, if any, to the normal performance in the current task.

\section{Spatial working memory error}

While the rats alternated between the two arms, a reentry into the arm visited in the previous trial was defined as SWM error (Olton et al., 1979). The rats with SAL injections made only one SWM error on average when the total number of SWM errors averaged over the $2 \mathrm{~d}$ of SAL injections were counted across the four different injection conditions (Fig. 5). The rats injected with MUS made one to two SWM errors on average in all conditions except for the bilateral HP condition. A paired $t$ test showed that the number of errors was significantly higher with the MUS injections into the hippocampus compared with the SAL injections into the same area $\left(t_{(15)}=3.83 ; p<0.01\right)$. There were no significant differences in SWM errors between SAL and MUS injections into the $\mathrm{PR}\left(t_{(15)}=0.17 ; p=0.8\right), \mathrm{HP} / \operatorname{conPR}\left(t_{(15)}=1.32\right.$; $p=0.2)$, or HP/ipsiPR $\left(t_{(15)}=1.04 ; p=0.3\right)$. An ANOVA with repeated measures also revealed a significant difference in SWM error among the four MUS injection conditions $\left(F_{(3,45)}=11.1\right.$; $p<0.001)$. Post hoc pairwise comparisons (Bonferroni's $t$ test) revealed that the bilateral hippocampal inactivations produced significantly more SWM errors than the other conditions (values of $p<0.05$ ). The results suggest that only the bilateral hippocampal inactivations impaired spatial information processing, but the unilateral inactivation of the hippocampus and bilateral/unilateral inactivations of the PR did not affect spatial cognition in the current task.

\section{Response bias}

Previously, we showed that the animals developed a perseverative, egocentric response strategy (i.e., making a stereotypic turn toward the food well on a particular side in the choice platform without considering the object associated with the food well) once they failed learning the current object-place pairedassociate task (Lee and Solivan, 2008, 2010; Jo and Lee, 2010). A response bias index was thus calculated to determine whether MUS inactivation also induced the response bias in the current study. The response bias index of 1 indicates a complete response bias to one direction and zero represents no response bias. Regardless of the injection sites, the rats injected with SAL consistently showed low response bias (Fig. 6), whereas the rats injected with MUS into all injection sites, except the HP/ipsiPR, displayed a strong preference to one side. An ANOVA with repeated measures revealed significant effects of drug injection day in the $\operatorname{HP}\left(F_{(3,21)}=15.6 ; p<0.001\right), \operatorname{PR}\left(F_{(3,21)}=23.6 ; p<0.001\right)$, and $\mathrm{HP} / \operatorname{conPR}\left(F_{(3,21)}=19 ; p<0.001\right)$. No effect was found in the HP/ispiPR $\left(F_{(3,21)}=2.65 ; p=0.07\right)$. Additional post hoc pairwise comparisons (Bonferroni's $t$ test) revealed increased response bias in MUS injection days of the HP (values of $p<0.05$ ), $\mathrm{PR}$ (values of $p<0.05$ ), and HP/conPR (values of $p<0.05$ ) relative to the response bias shown in the SAL injection days of each injection conditions.

\section{Simple object discrimination task}

To investigate whether the contralateral disconnection between the hippocampus and PR is also necessary for a simple object discrimination task in which no requirement is imposed for associating objects and their locations, the same rats were trained and tested for additional $6 \mathrm{~d}$ (Fig. 7). Two different sets of objects were assigned to arm 3 and arm 5 (Fig. 1C). The rats needed to choose a certain object in arm 3 (Boy) and arm 5 (Cylinder) regardless of the location of the rewarding objects within the choice platform. In this task, arm information was irrelevant because reward could be obtained solely on the basis of object identity information. The rats acquired the task quickly and reached performance criterion on the second day (Fig. 7). Then either SAL or MUS was injected into the HP/conPR. The animals exhibited normal performance with both SAL and MUS injections. An ANOVA with a repeated-measures design revealed no significant effect of drug injection day $\left(F_{(3,21)}=0.74 ; p=0.5\right)$, suggesting that the ipsilateral interactions between the hippocampus and $\mathrm{PR}$ are not necessary in a simple object discrimination task compared with the object-place paired-associate task.

\section{Discussion}

The current study investigated the functional interaction between the hippocampus and PR in object-place paired association. Bilateral infusions of MUS into either the hippocampus or $\mathrm{PR}$ in the object-place paired-associate task produced profound deficits in performance compared with the SAL injections, indicating that rats were unable to perform the task normally in the absence of the hippocampus or the PR. The MUS infusions into the hippocampus and PR across the contralateral hemispheres also resulted in severe impairment in performance, but the MUS injections into the hippocampus and PR in the same hemisphere did not affect the performance. These results suggest that the hippocampus and PR are both necessary because they communicate with each other to solve the current task. In contrast, in the simple object discrimination task, the animals were normal with the contralateral inactivations of the hippocampal-PR circuits. Also, PR inactivations in our study were never associated with pure spatial memory deficit. Overall, these results provide compelling evidence that the interaction between the hippocampus and PR is critical for object-place paired associations especially when the same objects appear in multiple locations with differential reward values.

Our laboratory has shown that the hippocampal lesions produce severe and irrecoverable impairment in the object-place 
paired-associate task similar to the one used in the current study (Lee and Solivan, 2008, 2010). When the role of the PR was further examined in the same task (Jo and Lee, 2010), rats with PR lesions were initially impaired in remembering familiar object-place paired associates but gradually improved their performance to normal levels. However, the same animals failed to learn new paired associates between novel objects and familiar places. The only difference between the task used in our previous studies and the current study is that rats were required to alternate between the two arms without entering the start box in the center platform in the current study. The goal of the current study was to recruit maximal interaction between the hippocampus and PR and the idea was that such interaction would occur more easily if more cognitive load is placed by requiring the animals to continuously process similar and ambiguous object-place associative events while alternating the two places (as opposed to entering an arm chosen by the experimenter as in our previous studies).

It is well known that the hippocampus is important for remembering spatial events (Scoville and Milner, 1957; O'Keefe and Nadel, 1978; Morris et al., 1982), and recent literature suggests that the PoR-MEC pathway provides spatial information to the hippocampus (Brun et al., 2002; Fyhn et al., 2004; Hargreaves et al., 2005; Knierim et al., 2006). It is not surprising, therefore, that the rats with hippocampal inactivations did not perform well in the current study because the animals needed to make a choice for an object on the basis of its associated location (Lee and Solivan, 2008, 2010). The fact that the hippocampalinactivated rats made more SWM errors (compared with the other drug injection conditions) by reentering the previously visited arm (Fig. 5) also supports the role of the hippocampus in spatial memory. However, our study suggests that sparing the hippocampus on one hemisphere is still sufficient for normal spatial information processing to occur.

With respect to the perirhinal cortical function, the literature suggests that the PR may not be critical for hippocampaldependent spatial tasks (Ennaceur et al., 1996; Bussey et al., 1999; Winters et al., 2004), although some studies suggest otherwise (Liu and Bilkey, 1998, 1999; Abe et al., 2009). In the current study, the PR manipulations (i.e., $\mathrm{PR}$ and $\mathrm{HP} /$ conPR) that produced severe impairment in performance (Figs. $3 A, 4 A$ ) were not accompanied by pure spatial memory deficits (Fig. 5). However, previous studies have demonstrated that the PR plays an essential role in processing object information (Buckley and Gaffan, 1997; Murray et al., 2000; Brown and Aggleton, 2001; Eacott et al., 2001; Bussey et al., 2003). In particular, these studies suggest that the PR lesions impair object discrimination when overlapping or similar stimuli were presented to induce feature ambiguity. It is possible that the two objects used in our object-place paired-associate task induced a high degree of ambiguity in object identity and maximally recruited the $P R$, because the objects were always presented simultaneously but were associated with different places with differential reward values across trials. Compared with the previous studies using object discrimination tasks, however, it is worth noting that the ambiguity in object identity was caused by its relationship to space in the current study.

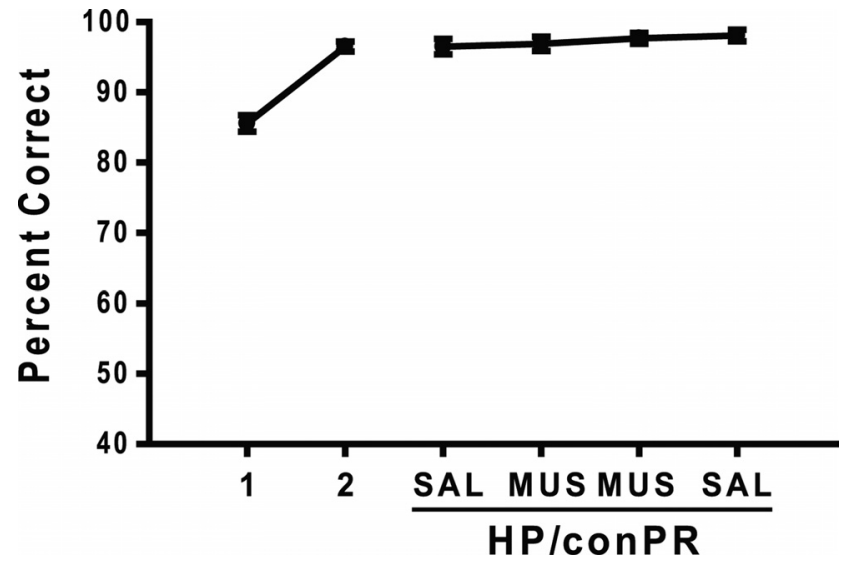

Figure 7. Learning and performance in the simple object discrimination task. Rats were trained for $2 \mathrm{~d}$ first and reached $\sim 95 \%$ performance level as a result. Neither SAL nor MUS injections into the $\mathrm{HP} /$ conPR afterward disrupted the performance. The graph shows mean \pm SEM.

The role of the EC (especially the LEC) may need to be considered when interpreting the results of the current study because, despite the existence of the reciprocal connections between the hippocampus and $\mathrm{PR}$, such connectivity is relatively weak compared with the heavy reciprocal connections between the hippocampus and EC (van Strien et al., 2009). Considering that the PR information is relayed to the hippocampus mostly via the LEC, it is possible that the behavioral deficits observed with the PR inactivations may have also affected the functional interaction between the LEC and hippocampus. Clarifying the source of behavioral deficits in the current study may require functional dissociations between the PR- and LEC-hippocampal connections. If the PR-to-LEC pathway to the hippocampus is limited to nonspatial information in the current study as shown in a foraging task (Hargreaves et al., 2005), it is possible that the function of that pathway is more related to object information only. In that case, it may be that disambiguation/identification of an object is performed sequentially from the PR to the LEC. In our previous study, rats with PR lesions relearned the object-place pairedassociate task, whereas they were unable to learn novel objectplace associations (Jo and Lee, 2010). This suggests that the PR performs a unique computation such as a more rudimentary feature conjunction/disambiguation (Bussey and Saksida, 2002) during the initial acquisition of the task, which may not be taken over by the LEC, whereas the LEC may extract object identity 
information afterward. If, however, the information processing in the PR and LEC is not limited to nonspatial information (allowing nonspatial-spatial interaction in those regions), more dynamic and complex mechanisms need to be considered. Heavy feedforward projections from the PoR to the PR and from the MEC to the LEC (van Strien et al., 2009) imply that this may well be the case. This scenario may require a revision of the prevalent theory that the nonspatial information and spatial information interact in the hippocampus after being processed through separate pathways in its upstream. It is important to note that more dynamic interactions with the hippocampal subfields are possible via the LEC than through the PR because the LEC has parallel connections with all three subfields of the hippocampus via the perforant paths, whereas the PR only connects to the CA1 (van Strien et al., 2009; Albasser et al., 2010).

In our disconnection paradigm, only the contralateral disconnection procedure between the hippocampus and PR was detrimental to the performance, and the ipsilateral disconnection procedure did not produce significant impairment. The results indicate that the ipsilateral communications between the hippocampus and PR (perhaps also involving LEC and MEC) is critical for processing object-place paired-associative representations and that the contralateral interactions between the two regions may not be sufficient. These results corroborate the anatomical findings that the PR shares dense connections with the ipsilateral CA1 and subiculum (Witter and Amaral, 2004). The same contralateral disconnection procedure, however, did not produce any deficit in the simple object discrimination task (Fig. 7 ), which is consistent with previous reports that simple object discrimination can be spared in the absence of either the hippocampus or the PR (Bussey et al., 2001; Eacott et al., 2001; Lee and Solivan, 2008, 2010; Jo and Lee, 2010). The results further support the idea that it is critical to require the animals to process both object and its associated spatial information at the same time to recruit a tight interaction between the hippocampus and PR.

The leading hypothesis suggests that object-place pairedassociative memory is likely to require unique contributions of multiple brain areas: The PoR-MEC pathway may process spatial information (Brun et al., 2002; Hargreaves et al., 2005), the PR-LEC may represent object information (Bussey et al., 2003; Albasser et al., 2010), and the spatial information may be further elaborated and associated with object information finally in the hippocampus (Gilbert and Kesner, 2004; Bachevalier and Nemanic, 2008; Lee and Solivan, 2008). Although additional experimental studies are needed in the future to verify such hypothesis and to measure detailed amounts of spatial-nonspatial interactions at different levels, the current study emphasizes that the functional interaction between the hippocampus and PR (directly and/or indirectly via the EC) is necessary when ambiguity in object identity needs to be cleared using spatial information.

\section{References}

Abe H, Ishida Y, Nonaka H, Iwasaki T (2009) Functional difference between rat perirhinal cortex and hippocampus in object and place discrimination tasks. Behav Brain Res 197:388-397.

Aggleton JP, Brown MW (2005) Contrasting hippocampal and perirhinal cortex function using immediate early gene imaging. Q J Exp Psychol B 58:218-233.

Aggleton JP, Kyd RJ, Bilkey DK (2004) When is the perirhinal cortex necessary for the performance of spatial memory tasks? Neurosci Biobehav Rev 28:611-624.

Albasser MM, Poirier GL, Aggleton JP (2010) Qualitatively different modes of perirhinal-hippocampal engagement when rats explore novel vs. familiar objects as revealed by c-Fos imaging. Eur J Neurosci 31:134-147.

Bachevalier J, Nemanic S (2008) Memory for spatial location and object- place associations are differently processed by the hippocampal formation, parahippocampal areas TH/TF and perirhinal cortex. Hippocampus 18:64-80.

Balderas I, Rodriguez-Ortiz CJ, Salgado-Tonda P, Chavez-Hurtado J, McGaugh JL, Bermudez-Rattoni F (2008) The consolidation of object and context recognition memory involve different regions of the temporal lobe. Learn Mem 15:618-624.

Brown MW, Aggleton JP (2001) Recognition memory: what are the roles of the perirhinal cortex and hippocampus? Nat Rev Neurosci 2:51-61.

Brun VH, Otnass MK, Molden S, Steffenach HA, Witter MP, Moser MB, Moser EI (2002) Place cells and place recognition maintained by direct entorhinal-hippocampal circuitry. Science 296:2243-2246.

Buckley MJ, Gaffan D (1997) Impairment of visual object-discrimination learning after perirhinal cortex ablation. Behav Neurosci 111:467-475.

Burwell RD (2000) The parahippocampal region: corticocortical connectivity. Ann N Y Acad Sci 911:25-42.

Burwell RD, Witter MP, Amaral DG (1995) Perirhinal and postrhinal cortices of the rat: a review of the neuroanatomical literature and comparison with findings from the monkey brain. Hippocampus 5:390-408.

Bussey TJ, Saksida LM (2002) The organization of visual object representations: a connectionist model of effects of lesions in perirhinal cortex. Eur J Neurosci 15:355-364.

Bussey TJ, Muir JL, Aggleton JP (1999) Functionally dissociating aspects of event memory: the effects of combined perirhinal and postrhinal cortex lesions on object and place memory in the rat. J Neurosci 19:495-502.

Bussey TJ, Dias R, Amin E, Muir JL, Aggleton JP (2001) Perirhinal cortex and place-object conditional learning in the rat. Behav Neurosci 115:776-785.

Bussey TJ, Saksida LM, Murray EA (2003) Impairments in visual discrimination after perirhinal cortex lesions: testing "declarative" vs. "perceptual-mnemonic" views of perirhinal cortex function. Eur J Neurosci 17:649-660.

Davachi L (2006) Item, context and relational episodic encoding in humans. Curr Opin Neurobiol 16:693-700.

Eacott MJ, Machin PE, Gaffan EA (2001) Elemental and configural visual discrimination learning following lesions to perirhinal cortex in the rat. Behav Brain Res 124:55-70.

Eichenbaum H, Lipton PA (2008) Towards a functional organization of the medial temporal lobe memory system: role of the parahippocampal and medial entorhinal cortical areas. Hippocampus 18:1314-1324.

Ennaceur A, Neave N, Aggleton JP (1996) Neurotoxic lesions of the perirhinal cortex do not mimic the behavioural effects of fornix transection in the rat. Behav Brain Res 80:9-25.

Fyhn M, Molden S, Witter MP, Moser EI, Moser MB (2004) Spatial representation in the entorhinal cortex. Science 305:1258-1264.

Gaffan D, Parker A (1996) Interaction of perirhinal cortex with the fornixfimbria: memory for objects and "object-in-place" memory. J Neurosci 16:5864-5869.

Gilbert PE, Kesner RP (2004) Memory for objects and their locations: the role of the hippocampus in retention of object-place associations. Neurobiol Learn Mem 81:39-45.

Hargreaves EL, Rao G, Lee I, Knierim JJ (2005) Major dissociation between medial and lateral entorhinal input to dorsal hippocampus. Science 308:1792-1794.

Jo YS, Lee I (2010) Perirhinal cortex is necessary for acquiring, but not for retrieving object-place paired association. Learn Mem 17:97-103.

Knierim JJ, Lee I, Hargreaves EL (2006) Hippocampal place cells: parallel input streams, subregional processing, and implications for episodic memory. Hippocampus 16:755-764.

Lee I, Solivan F (2008) The roles of the medial prefrontal cortex and hippocampus in a spatial paired-association task. Learn Mem 15:357-367.

Lee I, Solivan F (2010) Dentate gyrus is necessary for disambiguating similar object-place representations. Learn Mem 17:252-258.

Liu P, Bilkey DK (1998) Lesions of perirhinal cortex produce spatial memory deficits in the radial maze. Hippocampus 8:114-121.

Liu P, Bilkey DK (1999) The effect of excitotoxic lesions centered on the perirhinal cortex in two versions of the radial arm maze task. Behav Neurosci 113:672-682.

Morris RG, Garrud P, Rawlins JN, O’Keefe J (1982) Place navigation impaired in rats with hippocampal lesions. Nature 297:681-683.

Mumby DG (2001) Perspectives on object-recognition memory following 
hippocampal damage: lessons from studies in rats. Behav Brain Res 127:159-181.

Murray EA, Bussey TJ, Hampton RR, Saksida LM (2000) The parahippocampal region and object identification. Ann N Y Acad Sci 911:166-174.

O’Keefe J, Nadel L (1978) The hippocampus as a cognitive map. Oxford: Clarendon.

Olton DS, Becker JT, Handelmann GE (1979) Hippocampus, space, and memory. Behav Brain Res 2:313-365.

Saksida LM, Bussey TJ, Buckmaster CA, Murray EA (2006) No effect of hippocampal lesions on perirhinal cortex-dependent feature-ambiguous visual discriminations. Hippocampus 16:421-430.

Scoville WB, Milner B (1957) Loss of recent memory after bilateral hippocampal lesions. J Neurol Neurosurg Psychiatry 20:11-21.

van Strien NM, Cappaert NL, Witter MP (2009) The anatomy of memory: an interactive overview of the parahippocampal-hippocampal network. Nat Rev Neurosci 10:272-282.

Winters BD, Forwood SE, Cowell RA, Saksida LM, Bussey TJ (2004) Double dissociation between the effects of peri-postrhinal cortex and hippocampal lesions on tests of object recognition and spatial memory: heterogeneity of function within the temporal lobe. J Neurosci 24:5901-5908.

Witter MP, Amaral DG (2004) The hippocampal formation. In: The rat nervous system, Ed 3 (Paxinos G, ed), pp 635-704. Amsterdam: Elsevier. Witter MP, Groenewegen HJ, Lopes da Silva FH, Lohman AH (1989) Functional organization of the extrinsic and intrinsic circuitry of the parahippocampal region. Prog Neurobiol 33:161-253.

Witter MP, Wouterlood FG, Naber PA, Haeften TV (2000) Anatomical organization of the parahippocampal-hippocampal network. Ann N Y Acad Sci 911:1-24. 